\title{
GRB PROMPT EMISSION AND THE PHYSICS OF ULTRA-RELATIVISTIC OUTFLOWS
}

\author{
F. Daigne ${ }^{1}$
}

\begin{abstract}
Due to severe time constraints, the joint discussion for sessions I-IV has be been extremely short considering the number of hot topics related to GRB prompt emission studies and the physics of GRB ultra-relativistic outflows. I attempt to briefly describe the main issues raised in the talks and posters presented during the conference.
\end{abstract}

\section{Introduction}

A series of excellent talks in Session I (historical remarks) has clearly shown the continuous effort made by the community of high-energy astrophysics since the discovery of GRBs to characterize their prompt emission with an always improving time resolution and spectral coverage. The most recent progress are mostly due to the Swift and Fermi satellites. Thanks to Fermi/GBM, a large sample of long and short GRBs with good quality spectra from $8 \mathrm{keV}$ to $15 \mathrm{MeV}$ is now available, and in a few cases, the LAT extends this spectral coverage up to several GeV. This allows to discuss the spectral shape, including the possible appearance of several spectral components, in a much more robust way than before, and to confirm and extend many known results on the spectral evolution of bursts.

Since the discovery of GRB afterglows in 1997 and the confirmation of their cosmological origin, the standard theoretical framework for the prompt emission is internal dissipation within an ultra-relativistic outflow. The former is needed to reproduce the high variability of the light curves and the latter is related to the well-known compactness problem: a large Lorentz factor is needed to avoid a strong $\gamma \gamma$ annihilation. This framework is well established and accepted. However, the community is far from agreeing on the "details" of this scenario: mechanism for the acceleration of the outflow (thermal vs. magnetic), composition and geometry of the outflow (magnetization? thermal content? neutron content?), nature of the internal dissipation (photosphere vs. inernal shocks vs magnetic reconnection), nature of the radiative processes at work (comptonized thermal emission

${ }^{1}$ UPMC-CNRS, UMR 7095, Institut d'Astrophysique de Paris, 75014 Paris, France 
vs. synchrotron vs. SSC vs. ...). Expectedly, most of the open issues debated during sessions I-IV of the conference are somehow related to these fundamental questions.

\section{Agreeing on a list of key observations}

Review talks on prompt emission models often start with a list of "key observations that any model should reproduce". From the discussions during the conference, it clearly appears that a preliminary step is still needed: agreeing on this list.

- Distribution of spectral parameters in GRB prompt spectra. GRB spectral properties are at the heart of the debates on the origin of the prompt emission. However, the present status is not clear, at least for theorists. It is for instance often said that the distribution of peak energies is narrow, around a few $100 \mathrm{keV}$. This seems however in contradiction with most results presented during the conference which are based on the spectral analysis of Fermi bursts. The peak energy of the time-integrated spectrum varies a lot from a burst to another and peak energies well above $1 \mathrm{MeV}$ can be observed, which complement the fact, known since Beppo-SAX and HETE2, that low-peak energy bursts well below $100 \mathrm{keV}$ do exist. In addition, timedependent spectral analysis become more and more available and usually show large variations, over more than one decade, of the peak energy within a single burst. Therefore, the old idea of a narrow distribution of the peak energy must probably be given up. It remains that the true distribution of the spectral parameters is of the greatest interest to understand the prompt emission. What are the minimum and maximum peak energies which are observed in time-dependent spectra? Photospheric models may have difficulties to explain the lowest and highest values. What is the distribution of the low-energy photon index? Synchrotron models are known to face severe difficulties to reproduce the largest values $\alpha>-1$. Recently, a deviation from the standard Band shape has been found in several bright GBM bursts, some being presented during this conference. This deviation may be attributed to an additional component with a quasi-thermal shape. When the spectral analysis is made with such two components, the spectral parameters of the main one (Band) are affected. Then the impact of this multi-component analysis on the distribution of the spectral parameters of the non-thermal emission will have to be investigated in the future and may change our general view on this issue.

- Is there prompt GeV emission with an internal origin? It seems natural to associate the long-lasting emission above $100 \mathrm{MeV}$ detected by the LAT in a few bursts to the deceleration of the relativistic jet by its environment, as for the afterglow observed at lower frequencies. Going one step further, several authors proposed that the whole $\mathrm{GeV}$ emission (including the emission detected while the prompt emission in the GBM is still active) is due to the 
external shock, whereas it is widely accepted that the prompt soft gammaray emission has an internal origin. This leads to an interesting open issue which was briefly discussed during the conference: is it possible to prove that there is $\mathrm{GeV}$ emission of internal origin, at least in some bursts? Possible tests may be related to the characterization of the variability of the $\mathrm{GeV}$ lightcurve. Some participants suggested that the short spike seen at all energies in GRB 090926A already tells us the answer. This issue is of great importance, not only to distinguish between the internal and external origin of the high-energy emission, but also because $\mathrm{GeV}$ emission of internal origin would put a severe constraint on the radius of the emission site.

- Should we consider that the early steep decay seen by XRT is prompt highlatitude emission? Another key observation to understand the origin of the prompt emission is due to Swift/XRT. A large majority of bursts show an early steep decay in X-rays, which starts at the end of the prompt emission in the BAT, and decays with a steep temporal index close to -3 . The most natural explanation is the high-latitude emission from the prompt phase, which was predicted in 2002. Several groups have made a detailed comparison between the model and XRT data and shown an excellent agreement. If this interpretation is correct, it puts strong constraints on the value of the radius at the end of the prompt phase, which must be large.

\section{Observations}

Many new observational results have been presented during the conference and raised some interesting issues.

- Are the results of time-integrated/-dependent spectral analysis self-consistent? As illustrated by several contributions, time-dependent spectra are now obtained for an increasing number of bursts and can be compared to the timeintegrated spectrum which is always available. This leads to new issues, especially as most of the functionals used for the spectral analysis do not add up easily. The sum of two Band functions is not a Band function. The sum of several Band functions with a continuously evolving peak energy and intensity may also look like two smoothly connected power-laws, but the width of the transition around the break should increase when more Band functions are added, whereas this transition has a fixed width in the Band function. Therefore, the fact that the Band function is always used and seems to work well, both for the time-integrated and the time-dependent analysis with time bins of very different durations, is rather puzzling, especially as a strong spectral evolution is usually found within a given GRB. This issue is probably the most severe for the longest integration bins and one should question the information contained in the results of the timeintegrated spectral analysis.

- Hardness-intensity correlations: are they all self-consistent? This issue is obviously related to the previous one. Several correlations have been found 
between the hardness (measured by a hardness ratio, by the time-integrated peak energy or by the time-evolving peak energy) and the intensity (measured by the fluence or isotropic equivalent energy, by the peak flux or peak luminosity, or by the time-evolving flux or luminosity). For instance, a correlation between the time-dependent luminosity and the time-dependent peak energy has been recently found in a few Fermi burst and discussed during this conference. As the time-integration of such a correlation does not obviously give a new one between time-integrated quantities, the self-consistency between all these correlations should probably be studied, irrespective of the legitimate debate on possible selection biases.

- Multi-component spectra: what is the spectral evolution of each component? There is a growing interest for a multi-component spectral analysis of Fermi bursts, well illustrated during the conference: main non-thermal component (Band), possible additional quasi-thermal component at low energy, additional power-law-like component at high energy. Depending on the models, they may be associated to different radiation processes in the same region, or to different emission sites. The spectral evolution of each component may be the key to answer these questions and necessitates both an observational and theoretical effort, to characterize the observed evolution and to make predictions that can be compared to data.

- The shape of the additional component at high energy and the associated lowenergy excess. The spectral analysis of a few Femi/LAT bursts shows the need for an additional component, usually modeled by a single power-law, dominant at high energy (LAT range), and unexpectedly also at the lower end of the GBM spectral range (low-energy excess). Such a single powerlaw over $\sim 6$ decades is not easily reproduced by models, despite interesting attempts presented during the conference. However, a power-law is the simplest functional that can be used for spectral fits and it is probably only the quality of data that prevents better characterizing the spectral shape of this additional component. Any additional information of some possible curvature or cutoff, as in the only case GRB 090926A, would be valuable for the physical interpretation. A special interest should also be given to the associated low-energy excess: is it an X-ray component temporally correlated to the high-energy component or is it really a unique component over so many decades? It would be interesting to try to characterized this excess independently of LAT data, with the GBM or with an instrument having good X-ray capabilities, as MAXI whose results were presented during this conference.

- Can we expect a Swift+Fermi/LAT burst? As already mentioned, the physical origin of the $\mathrm{GeV}$ emission is even more puzzling than for the rest of the prompt emission, as both an internal and an external origin can be discussed. Part of the debate is related to the possibility of an early deceleration (before the end of the prompt phase). Unfortunately, early X-ray data are 
not available for LAT bursts, which would put interesting constraints on this scenario. It would be especially interesting to detect the XRT early steep decay, as - as discussed above - it can provide a precise information on the end of the prompt phase. Therefore, a burst with a simultaneous trigger by the LAT and by Swift would be of the greatest interest.

- Can we expect a Fermi/LAT burst with prompt optical data? Prompt optical detections remain rare but can provide important constraints on the prompt emission sites, as discussed during the conference. There are several models proposed to explain the few cases where such observations are available. Some of them predict a bright $\mathrm{GeV}$ emission (as the SSC model proposed for the naked eye burst). Therefore, a Fermi/LAT burst with prompt optical data would have a real interest. However, due to two independent low detection rates, such an observation seems even more challenging than the one discussed in the previous item.

\section{Models}

A general discussion of the three main GRB prompt emission models is out of the scope of this short contribution. I briefly mention the main open issues associated with each of them, as well as a few additional questions more related to the central engine and jet physics.

- Dissipative photospheres. This model has the best understood physics. Detailed calculations are already available and some of them have been presented during the conference. However, most of them focus on a "typical" spectrum. As discussed above, spectral evolution is also a key feature of GRBs. Therefore, more realistic calculations with predictions for this evolution within a pulse or a more complex light curve would allow to go one step further in the comparison with data. One issue is to understand if this model is compatible with the recent results of a multi-component spectral analysis. Another issue which has also been debated during the conference is the prediction for the polarization of the prompt emission. However, as also discussed in another session of this conference, it is not clear to know if data to compare are already available on this side. More generally, it is interesting to note that photospheric emission is almost unavoidable in all GRB models. The real issue is to understand if it can alone explain the whole prompt emission. The answer is partially related to some items discussed before, such as the existence of $\mathrm{GeV}$ emission of internal origin or the interpretation of the XRT early-steep decay. If photospheric emission has to be complemented by additional processes above the photosphere, one should understand which component of the spectrum has a photospheric origin and, from the shape of this component, if sub-photospheric dissipation is mandatory.

- Internal shocks. The main uncertainty of this model is related to the microphysics, which is usually parametrized by simple parameters $\left(\epsilon_{\mathrm{e}}, \epsilon_{\mathrm{B}}, \ldots\right)$ 
which are kept constant within a burst and can not be related yet to the shock conditions by physical prescriptions. The recent progress in the theory of shock acceleration have been obtained in the ultra-relativistic regime and the mildly relativistic regime relevant for internal shocks has not really been explored yet. On the other hand, using this simple parametrization, it is possible to model light curves and spectra produced by internal shock, and this model is probably, among the three main possibilities, the one that has been compared the more in detail with data, with promising results. It would therefore be of the greatest interest to learn more about the relevance of the assumptions made for the microphysics. Another well known issue of the model is the energetics. However, if the efficiency of internal shocks is known, the constraints on the efficiency of the prompt phase are less clear. They are usually based on simple versions of the afterglow model, that fails to reproduce the complex phenomenology of the early afterglow. When more elaborated models are used, including for instance a late injection to reproduce X-ray plateaus, it usually leads to so high efficiencies that they become challenging not only for internal shocks but for all other models. Progress in afterglow studies is clearly needed to better characterize this issue.

- Magnetic reconnection. This model is motivated by observational and theoretical works favoring a magnetic acceleration for GRB jets. It should however be remembered that, as illustrated by several contributions in this conference, magnetic acceleration of the jet does not necessarily mean a high magnetization $(\sigma \geq 1)$ at large distance where the prompt emission takes place. Magnetic reconnection can in principle solve many problems faced by the two previous models. On the other hand, the relevant physics is by far the least understood. Therefore, the main issue here is to know how to test the model and if detailed predictions for the temporal and spectral properties can be obtained for a comparison with data.

- Central engine and relativistic ejection. Several contributions have discussed the acceleration of the GRB outflow to relativistic speed and the early propagation of this jet, especially within the collapsing progenitor star in the case of long bursts. The relevant physics is very complex and in many cases, large simulations on super-computer are needed to address this problem in a realistic way. A clear difficulty is the lack of direct constraint, as the innermost region of the central engine is well below the photosphere and no light signal can be sent. If one focus on issues which are the most strongly connected to the prompt emission physics, one may list the two following questions: what is the final magnetization and thermal content at the end of the acceleration? What are the processes which govern the various timescales encountered in GRB light curves, from the shortest variability timescale to the duration of the prompt emission, including intermediate timescales such as pulse durations. We are probably still far from answering these two fundamental questions but the activity of the field has been beautifully illustrated during the conference with several convincing intermediate results. 
- Short vs. long bursts: where does the common physics start? Finally, the existence of several classes of GRBs remain to be understood. Several contributions have discussed the differences between short and long bursts, or non-collapsar and collapsar bursts, based on the host galaxies, the properties of the prompt emission, the distribution of $T_{90}$, etc.. On the theoretical point of view, it is usually assumed that short and long bursts differ by their progenitors and environment but that the same physics is at work. However, different progenitors may lead to different central engine and/or ejection mechanism and/or composition and geometry of the jet and/or internal dissipation mechanism,... With the recent progress in the observation of short GRBs, and especially the fact that some bright short GRBs can now be characterized with the same level of details than the long ones (temporal properties, spectral evolution), one may expect some progress in drawing the frontier between short and long bursts and understanding where the common physics start in these two classes of phenomena.

\section{Conclusion}

Forty five years after the discovery of GRBs, the understanding of the physical origin of the prompt emission remains a major challenge both on the observational and theoretical sides. As illustrated by the series of issues listed above, this is however a very active field of research with many stimulating discussions about various hot topics. Despite the difficulty, there is no doubt that new progress in the physics of the prompt emission and of GRB relativistic outflows will be achieved in the coming years. 
